\title{
Targeted knockout of the NUD gene in Siberian barley
}

Korotkova A.M. ${ }^{1 *}$, Kolosovskaya E.V. ${ }^{2}$, Gerasimova S.V. ${ }^{1,2}$, Hertig C. ${ }^{3}$, Otto I. ${ }^{3}$, Kumlehn J. ${ }^{3}$, Khlestkina E.K. ${ }^{4}$

${ }^{1}$ Kurchatov Genomic Center of the Institute of Cytology and Genetics, SB RAS, Novosibirsk, Russia

${ }^{2}$ Novosibirsk State University, Novosibirsk, Russia

${ }^{3}$ Leibniz Institute of Plant Genetics and Crop Plant Research (IPK) Gatersleben, Germany

${ }^{4}$ N.I. Vavilov All-Russian Institute of Plant Genetic Resources, St. Petersburg, Russia

*email: korotkova@bionet.nsc.ru

Barley (Hordeum vulgare L.) is an important food crop in the world and in Russia in particular. Naked barley provides some benefits in terms of human nutrition and health. The NUDUM (NUD) gene encodes a transcription factor involved in the formation of a lipidic layer at the grain surface of hulled barley. If the $N U D$ gene function is lost, lemma and palea do no longer adhere to the grain's pericarp, which entails the non-adherent hull (i.e. naked) phenotype. In a recent study, we obtained $n u d$ loss-of-function lines by sitedirected mutagenesis using customized RNA-guided Cas9 endonuclease in the spring barley cultivar 'Golden Promise'. It was demonstrated that the naked grain phenotype of these mutant lines is not accompanied by any modifications of agronomically relevant properties. The present investigation aims to apply the principle of converting hulled into naked barley in Siberian elite germplasm. To this end, a genetic engineering platform is being established that is applicable to a variety of genotypes including barleys from Russian breeders. As a methodical prerequisite, a panel of Russian barley cultivars was tested for the in vitro regeneration potential of immature embryo explants. The cultivars Aley, Biom and Signal performed best in this screen. Upon establishment of a protoplast transfection protocol, it was shown that mutations can be triggered in the NUD gene of $\mathrm{cv}$. Aley, thereby confirming that the used cas $9 / \mathrm{gRNA}$ vectors work effectively in this genetic background as well. Attempts to achieve site-directed mutagenesis at the wholeplant level using Agrobacterium-mediated and ballistic DNA transfer to embryo explants of cvs. Aley and Biom are in progress.

Acknowledgements: The study is supported by the Russian Science Foundation (project No. 21-66-00012) and by the Kurchatov Genomic Center of the Institute of Cytology and Genetics, SB RAS (075-15-2019-1662). 